\title{
Cubosomes: a vehicle for delivery of various therapeutic agents
}

\begin{abstract}
A drug delivery system is a formulation or device that safely brings a therapeutic agent to the specific body site at a certain rate to achieve an effective concentration at the site of drug action. To achieve therapeutic effects, it must be possible to load sufficient amounts of the active, which largely depends on the interaction of the vehicle and active. In this concern, cubosomes are bicontinuous cubic phase liquid crystals have many properties that make them appealing as a universal vehicle for drug delivery and it was considered as the drug nanocarrier due to their great potential as an alternative drug delivery system relative to liposome. Cubosomes are capable of loading lipophilic, hydrophilic, and amphiphilic drugs. This can be prepared by top-down or bottom-up approach. The main precursor of cubosome formation is monoolein whereas poloxamer 407 in a concentration range between $0 \%$ and $20 \% \mathrm{w} / \mathrm{w}$ with respect to the disperse phase use as a surfactant.
\end{abstract}

Volume 4 Issue | - 2018

\author{
Nilesh R Rarokar, Pramod B Khedekar \\ Department of Pharmaceutical Sciences, Rashtrasant Tukadoji \\ Maharaj Nagpur University, India

\begin{abstract}
Correspondence: Pramod B Khedekar, Nanotechnology Laboratory, Department of Pharmaceutical Sciences, Rashtrasant Tukadoji Maharaj Nagpur University, Nagpur, Maharashtra, India, Tel +9|7|22500324, Fax +9|7|22500355 Email nileshrarokar@outlook.com
\end{abstract}

Received: November 09, 2017 | Published: January 30, 2018

\section{Introduction}

A drug delivery system is a formulation or device that safely brings a therapeutic agent to the specific body site at a certain rate to achieve an effective concentration at the site of drug action. The release of drug in a predesigned manner is termed controlled drug release (CR), which is used to promote therapeutic benefits while minimizing toxic side-effects. Sustained release over an extended period of time may reduce the need for multiple dosing which will be a benefit in terms of reduced cost and increased patient compliance. The release rate of drugs must be controlled to achieve optimal drug release profiles. Surfactant and polymers are generally employed in the fabrication of controlled drug delivery systems because they form supra-assemblies, which are extensively exploited as active delivery vehicles. These systems include liquid crystalline aggregates or crosslinked gel networks that load, stabilize and eventually deliver active ingredients. Incorporation of drugs into the complex internal domains of these structures can facilitate diffusion controlled release of drug into the surrounding external aqueous environment. ${ }^{1-3}$ Providing new ways to modify pharmacokinetic profiles using lipid-based systems. ${ }^{4,5}$ To achieve therapeutic effects, it must be possible to load sufficient amounts of the active, which largely depends on the interaction of the vehicle and active. Further, the integrity of the active must be retained through all stages: preparation, storage, and use. The release rate of actives must be controlled to achieve optimal drug release profiles, while ease of preparation and vehicle stability must also be considered. An optimal delivery vehicle must successfully encompass all these properties.

\section{Cubosome}

Cubosomes are bicontinuous cubic phase liquid crystals have many properties that make them appealing as a universal vehicle for drug delivery. Cubosomes are nanoparticles, more accurately nanostructure particles, or self-assembled liquid crystalline particles with a solidlike rheology. However, the cubic phases are unique in that they possess very high solid-like viscosities because of their intriguing bicontinuous structures. Bicontinuous cubic liquid crystalline phase is an optically clear, very viscous material that has a unique structure at the nanometer scale. Its geometric model was supplied, prepared and examined for drug delivery. In the structure the surfactant assembles into bilayers that are twisted into a three dimension, periodic, minimal surface forming tightly packed structure, like "honeycombed" with bicontinuous domains of water and lipid. Cubosome particles are first prepared by mechanical fragmentation of the cubic lipid-water phase in a three-phase region containing a liposomal dispersion and to differentiate from liposome's, these particles have been termed as cubosomes. Its structure is different from liposome's because its structure can simultaneously accommodate water-soluble, lipidsoluble, and amphiphilic molecules. Three structure of cubosomes have been proposed Luzzati V et al., ${ }^{6}$ (i) P $n 3 m$ (D-surface) (Diamond surface), (ii) $\mathrm{I} a 3 d$ (G-surface) (Gyroid surface), and (iii) $\operatorname{Im} 3 \mathrm{~m}$ (P-surface) (Primitive surface), in terms of nodal surfaces. The structure generally maintains the efficacy; stability of actives such as vitamins and proteins. Cubosomes are thermodynamically stable; lasting indefinitely. Colloidal dispersions of cubosomes can be stabilized by the addition of polymers. They also possess the potential for controlled delivery of actives, where diffusion is governed by the tortuous diffusion of the active through the "regular" channel structure of the cubic phase. Cubosomes possess a sufficient average degree of molecular orientation in order to characterize by structural symmetry, and often form in aqueous surfactant system at relatively high ampiphile concentrations. In recent years, cubosomes (cubosome dispersions) were considered as the drug nanocarrier due to their great potential as an alternative drug delivery system relative to liposome. Cubosomes, especially made of binary systems, monoolein- water. That can self assemble into thermodynamically stable biocontinuous cubic liquid crystallize phases. ${ }^{8}$

\section{Advantages}

Cubosomes are capable of loading lipophilic, hydrophilic, and amphiphilic drugs. Because of the three-dimensional nanostructure with hydrophobic and hydrophilic domains, cubic liquid crystalline phases have been applied in pharmaceutical drug delivery. The large interfacial area can provide a complex diffusion pathway for sustained release of entrapped drug molecules, whereas lipid constituents are biocompatible, bioadhesive, and digestible., ${ }^{9,10}$ 


\section{Preparation}

Cubosomes usually have been produced by means of timeconsuming methods involving high-energy input. The dispersions were based either on glyceryl-monooleate/sunflower oil or glycerylmonooleate/retynilpalmitate mixtures plus a nonionic triblock polymer (Poloxamer 407) in water. Dispersions were produced by drop wise addition of a melt of lipids and poloxamer in water, followed by reduction of size by homogenization under high pressures at $80^{\circ} \mathrm{C}$. Recently the preparation and characterization of dispersions constituted of monoolein-rich monoglycerides with or without purified soya phospholipids. ${ }^{11}$ Dispersions were prepared by equilibration of the monoglyceride/phospholipid/ water cubic phase, subsequent fragmentation by a solution of Poloxamer 407, predispersing by probe sonication and finally high pressure homogenization. Moreover some authors have developed experimental protocols for cubosome production based on the use of organic solvents. Spicer and Hyden have proposed a method based on a dilution process of an ethanolic solution of monoolein with an aqueous solution of poloxamer. Ethanol was used as a hydrotrope to create a liquid precursor, spontaneously forming cubosomes after dilution. Finally Nakano et al. have suggested a method for the production of cubosomes based on hydration of a dry film of monoolein/poloxamer with an aqueous buffer. ${ }^{12}$

\section{Methods of preparation of cubosomes}

Three macroscopic forms of cubic phase are typically encountered; precursor, bulk gel and particulate dispersion. The precursor form exists as a solid or liquid material that forms cubic phase in response to a stimulus, such as contact with liquid. Bulk cubic phase gel is an optically isotropic, stiff, and solid like material in equilibrium with water can be dispersed into particles called cubosomes. The production of cubosomes entails two distinct technologies:

\section{Top-down technique}

Top-down approach begins with a suitable starting material and then sculpts the functionality from the material. The bulk cubic phase is first produced and then dispersed by high energy processing into cubosome nanoparticles. Bulk cubic phase resembles a clear rigid gel formed by water-swollen cross-linked polymer chains, but cubic phases differ in that they are a single thermodynamic phase and display periodic liquid crystalline structure. Cubic phases may behave as lamellar phases during dispersion with increasing shear: dispersed liquid crystalline particles form at intermediate shear rates, whereas a defect free bulk phase re-forms at higher shear rates. At high oscillatory frequencies, cubic phases become highly elastic. ${ }^{13}$

\section{Bottom-up technique}

The bottom-up approach first forms the nanostructure building blocks and then assembles them into the final material. It is more recently developed technique of cubosome formation, allowing cubosomes to form and crystallize from precursors on the molecular length scale. The formation of cubosomes by dispersion of inverse micellar phase droplets in water at $80^{\circ} \mathrm{C}$, then by slow cooling to allow the droplets to gradually crystallize into cubosomes. ${ }^{14}$

Dispersion of the nanoparticles produced in the cubosomes formation by several techniques

i. Sonication ii. High pressure homogenization

iii. Spontaneous emulsification

iv. Spray drying

v. Sonication and high-pressure.

Homogenization suggests the formation of complex dispersions containing vesicles and cubosomes with time-dependent ratios of each particle type. Coarse cubosomes on the micron scale possess the same D-surface cubic structure as their originating bulk cubic phase Spicer $\mathrm{PT}^{15}$ but after homogenization, the P-surface dominates, either because of the added polymer or other factors. ${ }^{16}$ Large-scale production of cubosomes and products containing them requires more robust processes. Smaller and more stable cubosomes are produced than those by high-energy processes, but some vesicles are also produced. A process was also developed to allow cubosome production from a powdered precursor. ${ }^{16}$ Spray-dried powders comprising monoolein coated with starch or dextran form cubosomes on simple hydration. The polymers immediately provide colloidal stabilization of the cubosomes.

\section{Material used in cubosomes formation}

Bicontinuous cubic phases are found in natural lipids, cationic Boretta $\mathrm{M}^{17}$ and nonionic surfactants Lynch $\mathrm{ML}^{18}$ and polymer systems, although the lipid most widely used to construct bicontinuous cubic phases is the monoglyceride monoolein, monoglycerides spontaneously form bicontinuous cubic phases upon the addition of water, are relatively insoluble, and are resistant to changes in temperature. The main precursor of cubosome formation is monoolein. Monoolein or glyceryl monooleate is a mixture of the glycerides of oleic acid and other fatty acids, consisting mainly of the monooleate. ${ }^{19-21}$ The glycerol moiety may form hydrogen bonds with water in an aqueous environment and is commonly referred to as the head group. The hydrocarbon chain gives hydrophobic characteristics to monoolein and is often termed the tail. Commercially available monoolein may be obtained in two forms, a mixed glyceride form or as distilled monoolein; the distilled monoolein is preferred for pharmaceutical applications because of its high purity. It swells in water, giving rise to several lyotropic liquid crystalline structures.

When lipid molecule is heated, instead of melting directly convert into an isotropic liquid. Surfactants, which are used in the production of cubosomes, are poloxamer 407 in a concentration range between $0 \%$ and $20 \% \mathrm{w} / \mathrm{w}$ with respect to the disperse phase. The concentration of the monoglyceride/surfactant mixture generally takes between $2.5 \%$ and $10 \% \mathrm{w} / \mathrm{w}$ with respect to the total weight of the dispersion. Polyvinyl alcohol (PVA) used in addition to poloxamer as a stabilizing agent of the dispersion.

\section{Application of cubosome}

a) Formulations of control release drug delivery system of solubilize substances.

b) Cubic phase is more applicable for control release because of;

c) Its small pore size

d) Ability to solubilize hydrophilic, hydrophobic and amphiphilic molecules 
e) Its biodegradability by simple enzyme

f) Widely used in cancer therapy

g) Used in topical, mucosal deposition and delivery of different drugs

h) The properties of bioadhesion and penetration enhancement of cubosomes suggest their potential utility in skin cancer (e.g., melanoma) treatment, there is currently no formulation addressing this need. Moreover, there is emerging interest in using statistical methods to optimize pharmaceutical formulations. ${ }^{22-25}$

A short list of applications includes the delivery of actives for periodontal disease and implants via in vivo and topical delivery, and as bioadhesives.

\section{Conclusion}

Cubosome have great potential to use as a vehicle for drug delivery of various therapeutic agents.

\section{Acknowledgements}

None.

\section{Conflicts of interest}

The author declares no conflict of interest.

\section{References}

1. Shah JC, Sadhale Y, Chilukuri DM. Cubic phase gels as drug delivery systems. Adv Drug Deliver Rev. 2001;47(2):229-250.

2. Burrows R, Collett JH, Attwood D. The release of drugs from monoglyceride-water liquid crystalline phases. Int $J$ Pharm. 1994;111(3):283-293.

3. Drummond CJ, Fong C. Surfactant self assembly objects as novel drug delivery vehicles. Current Openion Colloid Interface Science. 2000;4(6):449-456

4. Fong W, Hanley T, Boyd B. Stimuli responsive liquid crystals provide 'on-demand' drug delivery in vitro and in vivo. J Control Release. 2009;135(3):218-226.

5. Dong YD, Larson I, Hanley T, et al. Bulk and dispersed aqueous phase behavior of phytantriol: effect of vitamin $\mathrm{E}$ acetate and $\mathrm{F} 127$ polymer on liquid crystal nanostructure. Langmuir. 2006;22(23):9512-9518.

6. Luzzati V, Tardieu A, Gulik Kryzwicki T, et al. X-ray study of model systems: structure of the lipid-water phases in correlation with the chemical composition of the lipids. Nature. 1968;51(50:37-43.

7. Bei D, Marszalek J, Youan BC. Formulation of Dacarbazine Loaded Cubosomes-Part I: Influence of Formulation Variables. AAPS Pharm Sci Tech. 2009;10(3):1032-1039.

8. Fontell K, Mandell L, Ekwall P. Isotropic Mesophases in Systems Containing Amphiphilic Compounds. Acta Chem Scand. 1968;22(1968):3209-3223.
9. Barauskas J, Johnsson M, Joabsson F, et al. Cubic phase nanoparticles (cubosome): principles for controlling size, structure, and stability. Langmuir. 2005;21(6):2569-2577.

10. Barauskas J, Johnsson M, Tiberg F. Self-assembled lipid superstructures: beyond vesicles and liposomes. Nano Lett. 2000;5(8):1615-1619.

11. Esposito E, Eblovi N, Rasi S, et al. Lipid based supramolecular system for topical application: A Preformulatory study. AAPS Pharm Sci Tech. 2003;5(4):1-15.

12. Nakano M, Sugita A, Matsuoka H, et al. Small angle $\mathrm{x}$-ray scattering and 13C NMR investigation on the internal structure of cubosomes. Langmuir. 2001;17(13):3917-3922.

13. Radiman S, Toprakcioglu C, Mcleish T. Rheological study of ternary cubic phases. Langmuir. 1994;10(1):61-67.

14. Almgren M, Edwards K, Gustafsson J. Cryotransmission electron microscopy of thin vitrified samples. Curr Opin Colloid Interface Sci. 1996;1(2):270-278.

15. Siekmann B, Bunjes H, Koch MHJ, et al. Preparation and structural investigations of colloidal dispersions prepared from cubic monoglyceride-water phases. Int J Pharm. 2002;244(1-2):33-43.

16. Spicer PT, Small WB, Lynch ML, et al. Dry powder precursors of cubic liquid crystalline nanoparticles (cubosomes). Journal of Nanoparticle Research. 2002;4(4):297-311.

17. Boretta M, Cantu L, Corti M, et al. Cubic phases of gangliosides in water: Possible role of the conformational bistability of the headgroup. Physica A. 1997;236(1-2):162-176.

18. Lynch ML, Kochvar KK, Burns JL, et al. Langmuir. 2000;16(7):3537.

19. Guerrero QD, Buri P. Monoolein: A Review of the Pharmaceutical Applications. Drug Development and Industrial Pharmacy. 2000;26(8):809-820.

20. Shanmugam T, Banerjee R. Nanostructured self assembled lipid materials for drug delivery and tissue engineering. Ther Deliv. 2011;2(11):1485-1516.

21. Moquin PHL, Temelli F. Production of monoolein from oleicacid and glycerol in supercritical carbon dioxide media: A kinetic approach. The Journal of Supercritical Fluids. 2008;44(1):40-47.

22. Solanki AB, Parikh JR, Parikh RH. Formulation and optimization of piroxicam proniosomes by 3-factor, 3-level Box-Behnken design. AAPS PharmSciTech. 2007;8(4):1-86.

23. Maghsoudi A, Shojaosadati SA, Vasheghani Farahani E. 5hFluorouracilloaded BSA nanoparticles: formulation optimization and in-vitro release study. AAPS Pharm Sci Tech. 2008;9(4):1092-1096.

24. Nattapulwat N, Purkkao N, Suwithayapan O. Preparation and application of carboxymethyl yam (Dioscorea esculenta) starch. AAPS Pharm Sci Tech. 2009;10(1):193-198.

25. Rarokar NR, Saoji SD, Raut NA, et al. Nanostructured Cubosomes in a Thermoresponsive Depot System: An Alternative Approach for the Controlled Delivery of Docetaxel. AAPS PharmSciTech. 2015;17(2):436-445. 